\title{
OPEN A serological assay to detect SARS-CoV-2 antibodies in at-home collected finger-prick dried blood spots
}

\author{
Donna Grace Karp, Kenneth Danh, Noemi Fonseca Espinoza, David Seftel, \\ Peter V. Robinson ${ }^{\bowtie}$ Cheng-ting Tsai ${ }^{\bowtie}$
}

Accurate surveillance of coronavirus disease 2019 (COVID-19) incidence requires large-scale testing of the population. Current testing methods require in-person collection of biospecimens by a healthcare worker, limiting access of individuals who do not have access to testing facilities while placing both patients and healthcare workers at risk of exposure to infection. We report the development and validation of a at-home finger-prick dried blood spot collection kit and an analysis method. We demonstrated $100 \%$ sensitivity and specificity using at-home collected specimens across the US. Such methods may facilitate the conduct of unbiased serosurveys within hard to reach populations and help reduce the sample collection burden of serological testing on both health care systems and individuals alike.

Severe acute respiratory syndrome coronavirus 2 (SARS-CoV-2), formerly known as 2019-nCoV, is an enveloped, single-stranded RNA virus in the family Coronaviridae, genus Betacoronavirus ${ }^{1-3}$. At the time of writing, SARS-CoV-2 pandemic has led to more than 35 million confirmed cases globally and claimed approximately 1 million lives as of Oct 6th, 2020.

While direct detection of the virus by RNA or antigen assays remains the standard for confirming acute SARS-CoV-2 infection, serological antibody testing is of increasing importance for many reasons ${ }^{4-8}$. Serological assays are important for assessing asymptomatic infection in close contacts, identifying individuals with resolved infection as potential contributors of convalescent plasma therapy, enabling estimation of population infection prevalence and illuminating our understanding of contributing factors conferring immunity to re-infection.

All currently approved serological tests require blood specimens collected in-person by a healthcare worker either through phlebotomy or finger-prick. The need for in-person collection limits access to testing for individuals who may not be able to get to a testing center due to distance, disability or inconvenience. The in-person collection process is labor intensive, driving up testing costs and diverting healthcare resources from the care of ill individuals. In-person collection processes carry an increased risk of infection due to potential exposure to a confluence of contagious individuals and consumes large quantities of valuable personal protective equipment (PPE).

In principle, rapid tests such as lateral flow assays may at some point be approved for at-home testing. However, lateral flow tests have shown an alarmingly wide performance dispersion even when performed by trained personnel in controlled clinical settings, with many tests exhibiting inadequate sensitivity and specificity ${ }^{9}$. The accuracy of lateral flow tests for at-home testing will require significant improvement and extensive additional validation before they can be trusted as clinically and epidemiologically useful. Integrity in self-reporting of the results of home-administered lateral flow tests also poses major challenges.

Here, we report the development of an at-home finger-prick dried blood spot collection kit and an accompanying laboratory test to elute and accurately detect antibodies in this convenient sample type with $100 \%$ concordance to their clinical status. For this study, we created standardized dried blood spot collection kits for reciprocal mailing to and from the homes of potential participants. These kits contained lancets for self-collection of blood and dried blood spot cards to capture and preserve the specimens for transport. The kits were then mailed back to a central laboratory for extraction and analysis. This type of home-to-lab sourced samples can 


\begin{tabular}{|c|c|c|}
\hline & COVID-19 & Healthy control \\
\hline Number of donors & 31 & 80 \\
\hline Gender & 20 Female & 48 Female \\
\hline Age (years) & $24-86$ & $25-75$ \\
\hline \multicolumn{3}{|c|}{ Distance to enable biosciences lab (miles) } \\
\hline$<100$ & 20 & 66 \\
\hline $100-1500$ & 0 & 5 \\
\hline$>1500$ & 11 & 9 \\
\hline \multicolumn{3}{|l|}{ Laboratory confirmation } \\
\hline PCR & 30 & 0 \\
\hline Serum/plasma antibody test & 1 & 4 \\
\hline
\end{tabular}

Table 1. Study participant demographics.

provide the high quality and consistency of a reference lab based serological test without exposing patients or healthcare workers to unnecessary risks.

\section{Results}

Cohort characteristics. We used social media (e.g. Facebook, Twitter, Medium) and community referrals to recruit volunteers for the study. We aimed to enroll both COVID-19 patients (positive controls) and healthy donors (negative controls) to validate the sensitivity and specificity of the serological test using self-collected dried blood spots. The COVID-19 patients were eligible to participate in the study if they presented with COVID-19 symptoms and were confirmed positive by either RT-PCR for SARS-CoV-2 RNA and/or a validated serum/plasma serological tests for SARS-CoV-2 antibodies. The control patients were included in the study if they had no symptoms and/or no known exposure to SARS-CoV-2 and/or tested negative by validated serum/ plasma serological tests for SARS-CoV-2 antibodies.

Eligible participants received a dried blood spot mailer kit that included comprehensive instructions for self-collection of finger-prick spots. The mailer kit contained a pre-printed return label to ship the kit back to the Enable Biosciences laboratory in South San Francisco by USPS.

A total of 111 specimens were received for the study, where 31 were from COVID-19 patients and 80 were from healthy donors. The patient demographics were summarized in Table 1. Notably, several donors were from out-of-state locations distant from the Enable Biosciences facilities. Since the dried blood spots were returned via ground shipping at ambient temperature, the inclusion of such donors allowed us to investigate the potential impact of long-distance transportation on sample quality and analytical performance.

Detection of SARS-CoV-2 antibody in self-collected finger-prick dried blood spots. We first evaluated the clinical sensitivity and specificity of self-collected finger-prick dried blood spots. Among 31 COVID-19 patients, 31 tested positive for SARS-CoV-2 antibodies against S1 protein. For 80 healthy donors, 80 tested negative for SARS-CoV-2 antibodies. The preliminary results indicated $100 \%$ sensitivity (95\% CI $89-100 \%)$ and $100 \%$ specificity (95\% CI 95-100\%) of mailer-based finger-prick dried blood spot specimens (Fig. 1). The corresponding positive predictive value (PPV) and negative predictive value were $100 \%$. Future studies could affirm the observed results at a larger scale.

To further affirm the observed signals were indeed specific for the SARS-CoV-2 antibodies, we also re-analyzed the samples using conjugates made of non-SARS-CoV-2 protein. As expected, no signals were observed for both COVID19 patients and healthy controls specimens (Supplementary Figure S1). Additionally, we also performed protein $\mathrm{G}$ beads pull down experiment to remove immunoglobulins from the samples, and we observed substantial loss of signals from the COVID19 patient specimens (Supplementary Figure S2). Taken together, the data supports the notion that the dried blood spot assay is specifically responsive to SARS-CoV-2 antibodies.

In addition to clinical performance, we also evaluated the signal distribution between samples collected in immediate proximity to the testing laboratory and those from distant areas. For COVID-19 patients, 20 specimens were collected within 100 miles of testing labs, while 11 specimens were collected from locations over 1500 miles away. The signal distribution between these two groups did not reach statistical significance (Supplementary Figure S3). Similar observations were made for the healthy control patient groups. The actual transit time of the completed kits, irrespective of the individual's physical distance away from the lab, ranged from 0 to 5 days. $88 \%$ of all test kits arrived within 3 days of the person mailing the completed kit back to Enable Biosciences using either USPS Priority or First-Class Mail. The data demonstrated excellent dried blood spot specimen integrity even after long-range transportation at ambient temperatures.

Considering the study was conducted in spring where the temperature profile is mild across the US, we further performed a simulated transport analysis. Contrived dried blood spot specimen (one with signal substantially above the assay cutoff, one with signal slightly above the cutoff and the other with signal below the cutoff) were subjected temperature cycling to mimic summer and winter conditions (Supplementary Tables S4 and S5). The results showed that the signals were comparable between summer/winter cycling and without cycling (Table 2). All but one positive specimen remained positive and all negative specimen remained negative, affirming the 


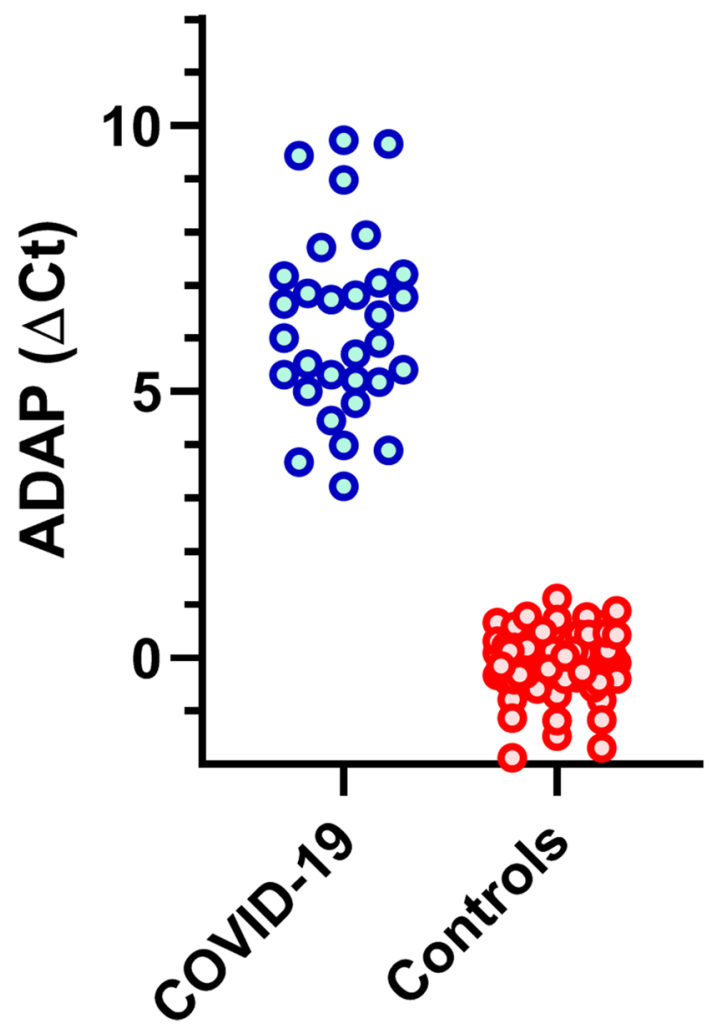

Figure 1. SARS-CoV-2 antibodies levels in self-collected finger-prick dried blood spots. Dried blood spot eluents were tested by the ADAP method for antibodies against the S1 protein of SARS-CoV-2. Signals were coded blue for COVID-19 and red for control donors. The $y$-axis is the signal output from ADAP, calculated by subtracting the Ct value of the specimen to the blank control (buffer C).

\begin{tabular}{|l|l|l|l|l|}
\hline Sample group & Test point & $\mathbf{N}$ & $\mathbf{\Delta C t}(\mathbf{S D})$ & Positive (\%) \\
\hline \multirow{4}{*}{ Negative } & $\mathrm{T}=0$ & 10 & $0.44(0.24)$ & $0(0)$ \\
\cline { 2 - 5 } & Summer & 10 & $0.25(0.52)$ & $0(0)$ \\
\cline { 2 - 5 } & Winter & 10 & $0.16(0.34)$ & $0(0)$ \\
\hline \multirow{4}{*}{ Mow positive } & $\mathrm{T}=0$ & 20 & $4.52(0.46)$ & $20(100)$ \\
\cline { 2 - 5 } & Summer & 20 & $4.12(0.40)$ & $19(95)$ \\
\cline { 2 - 5 } & Winter & 20 & $4.23(0.24)$ & $20(100)$ \\
\hline \multirow{3}{*}{ Medium positive } & $\mathrm{T}=0$ & 20 & $7.08(0.44)$ & $20(100)$ \\
\cline { 2 - 5 } & Summer & 20 & $6.51(0.45)$ & $20(100)$ \\
\cline { 2 - 5 } & Winter & 20 & $6.86(0.26)$ & $20(100)$ \\
\hline
\end{tabular}

Table 2. ADAP SARS-CoV-2 antibody signals before and after simulated transport. Sample panels composed of ten replicates of negative dried blood spot, 20 replicates of low positive dried blood spot and 20 replicates of medium positive dried blood spot were subjected to simulated summer and winter shipping conditions (specified in Supplementary Tables S4 and S5). The average and standard deviations of $\Delta$ Ct were reported. In addition, the number of positive samples (and the corresponding percentage) were also reported.

stability of dried blood spot specimen under extreme conditions. Furthermore, we also demonstrated that the eluent were stable for at least 1 week in $-80^{\circ} \mathrm{C}$ (Supplementary Figure S6), allowing the laboratory to conduct elution and testing on different days.

While the study was primarily conducted with ADAP assay for detection of SARS-CoV-2 antibodies against the $\mathrm{S} 1$ protein, it could be valuable to detect both $\mathrm{S} 1$ and nucleocapsid $(\mathrm{N})$ protein antibodies in a single assay. To that end, we analyzed a portion of the specimens with sufficient leftover eluent volumes for these two antibody targets (Supplementary Figure S7). Indeed, all of the S1 antibody positive patients also have N protein antibodies, affirming the multiplex detection capability of ADAP. 


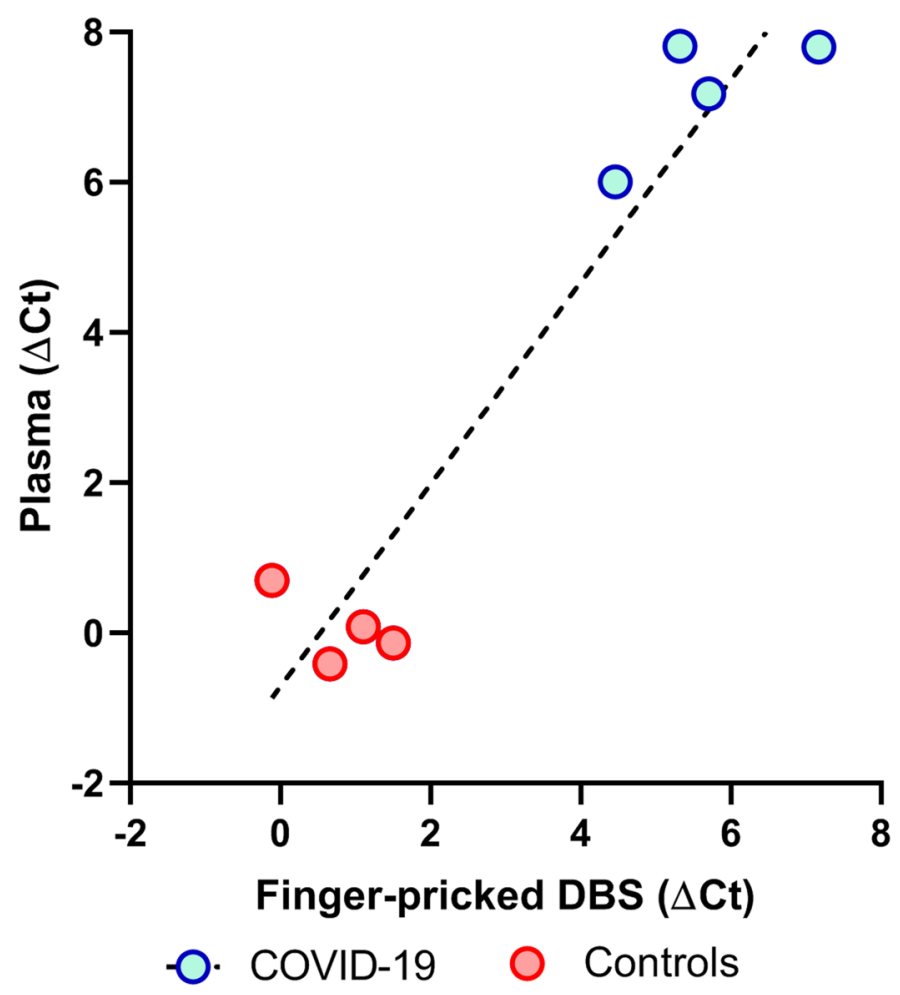

Figure 2. Correlation of signals between self-collected finger-prick dried blood spots and venipuncture plasma samples $(R=0.96)$. Self-collected mail in dried blood spot specimens from COVID-19 $(n=4)$ and healthy controls $(n=4)$ were analyzed in comparison to venipuncture plasma samples from the same individuals.

Correlation of SARS-CoV-2 antibody signals between sample types. In addition to sensitivity and specificity, it is critical to compare the antibody signals in self-collected finger-prick dried blood spots to venipuncture-based blood samples. The lancets used in self-collected finger-prick samples are shorter than the needles used for venipuncture. Untrained individuals collecting at home may use a wide range of lancing techniques and differing puncture sites. As a result, finger-prick blood could conceivably be partially diluted by extra-venous tissue fluids.

To evaluate the impact of these possibilities, we collected K2-EDTA plasma samples by standard phlebotomy from 4 COVID-19 patients and four healthy controls. The plasma samples were tested using the standard ADAP assay for SARS-CoV-2 antibodies. The data showed that the plasma signals were highly consistent and well correlated with those from self-collected sample types (Fig. 2), effectively eliminating the theoretical sampling concerns of at-home collected dried blood spot samples for SARS-CoV-2 antibody measurement.

\section{Discussion}

Serological testing plays a significant role in the management of SARS-CoV-2 infection. First, the presence of antibodies indicates past exposure to SARS-CoV-2 for both asymptomatic and ill patients. Thus, antibody-based serosurveys are key to measuring disease prevalence at the population level. This data is critical for accurate characterization of essential parameters such as fatality rates, basic reproduction number (Ro) and others ${ }^{10}$. Secondly, convalescent plasma has been shown as a safe and promising therapy to mitigate disease ${ }^{11,12}$. Serological testing can help identify eligible donors for further antibody characterization by methods such as neutralization assays.

However, the constraint of phlebotomy for sample collection for current serological tests has curtailed these potential benefits. Many at-home collection RT-PCR RNA tests have received regulatory approvals. To yield a complete picture of SARS-CoV-2 infection, it is highly desirable to pair suitable molecular diagnostic with selfcollected serological tests.

The dried blood spot collection kit and testing method reported in this study have the potential for widespread use. Obviating phlebotomy could improve both testing safety and testing access, while reducing the sample collection burden on severely stressed healthcare systems. The stability of dried blood spots at ambient temperature for extended time periods permits standard mail parcel transportation service use without requiring cold chain preservation measures. Importantly, mailer-based self-collected samples may reduce bias and improve participation within underserved or sequestered communities by reaching populations beyond those accessed by serosurveys that rely solely on collection at drive-through clinics.

Since the dried blood spot modality has the potential for wide scale screening, it is critical to ensure the specificity of this test. The use of ADAP methods for dried blood spot eluent testing may have an additional unique advantage in that the ADAP method consumes a very small volume of eluent (e.g. $8 \mu \mathrm{L})$ per test. It is 
thus possible to conserve samples to support further analyses. For instance, it is potentially feasible to conduct the primary screening of the eluent by detecting only anti-S1 protein antibodies. Samples that test positive can then be confirmed by anti-N protein antibody test without having to elute additional samples from another spot.

In summary, we report a self-collection kit for at-home finger-prick dried blood spot collection with a method to elute and test the specimen that show comparable analytical performance to venipuncture-derived blood samples. If proven successful at large scale, this method can greatly facilitate the conduct of unbiased serosurveys within hard to reach populations and help reduce the sample collection burden of serological testing on both health care systems and individuals alike.

\begin{abstract}
Methods
Materials. The SARS-CoV-2 spike protein (S1) containing amino acids 1-674 with an Fc-tag at the C-terminus (\#31806), expressed in HEK293 cells and SARS-CoV-2 nucleocapsid (N) protein expressed in E. coli were purchased from the Native Antigen Company (Oxford, United Kingdom). Oligonucleotides used in the study were custom ordered from Integrated DNA Technologies (Coralville, IA). Platinum Taq polymerase (\#10966026), SYBR qPCR 2X master mix (\#4385610), Dithiothreitol (DTT \#202090) and sulfo-SMCC (\#22122) were purchased from Thermo Fisher (Waltham, MA). DNA ligase (\#A8101) was purchased from LGC (Teddington, United Kingdom). Other reagents are detailed in the method sections as appropriate.
\end{abstract}

Human specimens used in the study. All clinical samples were obtained with written informed consent, and the study design was approved by the Institutional Review Board of Western IRB, which is accredited by the Association for the Accreditation of Human Research Protection Programs (AAHRPP) under applicable laws and regulations (IRB No. 20180015). All the test processes were performed in accordance with the relevant guidelines and regulations. Individuals who tested positive for SARS-CoV-2 RNA by RT-PCR between March and April 2020 were recruited to the study via social media. Healthy donors without SARS-CoV-2 symptoms were enrolled as negative controls. Dried blood spot collection kits were mailed to each participant for at-home sample collection. We requested two to five dried blood spots from each participant. Completed dried blood spots were dropped off in standard United States Postal Service (USPS) blue drop boxes for ground return shipment to Enable Biosciences. The entire process was done at ambient temperature without a cold chain.

A total of 111 self-collected finger-prick dried blood spot specimens were received. Negative control specimens were collected from 80 donors, and positive specimens were collected from 31 COVID-19 patients. The patients were deemed infected if symptomatic and tested positive by RT-PCR $(\mathrm{N}=30)$ or by validated serum antibody tests $(\mathrm{N}=1)$.

In addition to dried blood spots, K2-EDTA plasma specimens were collected by phlebotomy from eight selected participants (four from COVID-19 patients and four from healthy controls).

Dried blood spot collection kit. The dried blood spot collection kit contained a sample collection instruction sheet, disposable lancets, a paper card, alcohol pads, Band Aids and a pre-printed return label. The participant was asked to clean the collection site with the sterile alcohol pad and then use the disposable lancet to prick the finger. Finger-prick blood was then carefully dropped onto each dried blood spot card until a complete circle was filled. A minimum of two spots per participant was requested. The kit contained multiple lancets in case a single prick proved insufficient to generate multiple spots. Given that the SARS-CoV-2 virus is rarely detected in the blood of recovered donors or donors with mild to moderate symptoms, the dried blood spot specimens presented a minimal biohazard risk ${ }^{13,14}$. Furthermore, dried blood spots were fully dried prior to shipment, thus further lowering the risk profile. The dried blood spot return kits were transported by standard postal mail service with UN3373 Biological Substance Category B Labels affixed. Once received, the dried blood spot cards were stored at $2-8{ }^{\circ} \mathrm{C}$ until analyzed.

Synthesis of protein-DNA conjugates for SARS-CoV-2 antibody assays. The synthesis of SARSCoV-2 protein DNA conjugates and DNA sequences used had been described previously ${ }^{15}$. Briefly, for SARSCoV-2 spike protein S1 subunit-DNA conjugates, the proteins were buffer exchanged in reaction buffers ( $55 \mathrm{mM}$ sodium phosphate, $150 \mathrm{mM}$ sodium chloride, $20 \mathrm{mM}$ EDTA, $\mathrm{pH} 7.2$ ) to make $1 \mathrm{mg} / \mathrm{mL}$ solutions. A $1 \mu \mathrm{L}$ solution of $8 \mathrm{mM}$ sulfo-SMCC was added to $10 \mu \mathrm{L}$ of each protein solution. The reaction mixtures were incubated at room temperature for $2 \mathrm{~h}$. Thiolated DNA was suspended in reaction buffers to $100 \mu \mathrm{M}$. A $3 \mu \mathrm{L}$ solution of thiolated DNA solution and $4 \mu \mathrm{L}$ of $100 \mathrm{mM}$ solution of DTT were mixed to reduce dimerized thiolated DNA to monomer forms. The solution was then incubated at $37^{\circ} \mathrm{C}$ for $1 \mathrm{~h}$. The excess sulfo-SMCC in protein mixtures and DTT in thiolated-DNA were removed by $7 \mathrm{~K}$ MWCO Zeba spin column (Thermo Fischer, Waltham, MA). The thiolated DNA and protein solutions were then pooled and incubated overnight at $4{ }^{\circ} \mathrm{C}$. Finally, proteinDNA conjugates were purified by $30 \mathrm{kDa}$ MWCO filter (Millipore, Burlington, MA). Conjugate concentrations were determined by BCA assay (Thermo Fischer). Conjugation efficiencies were analyzed by SDS-PAGE and silver staining as described previously ${ }^{15-18}$. DNA-to-protein ratios of the conjugates were estimated by UV-VIS absorption and typically fell in the range of 2-to-1. Protein-DNA conjugates were stored at $4{ }^{\circ} \mathrm{C}$ for short-term usage or aliquoted for long-term storage at $-80^{\circ} \mathrm{C}$. The synthesis of SARS-CoV-2 nucleocapsid proteins were similar to the procedure above, except the use of nucleocapsid protein in lieu of spike protein.

Detection of SARS-CoV-2 antibodies in dried blood spots. The dried blood spot analysis comprised elution and testing steps. For elution, six $3 \mathrm{~mm}$ discs were punched from a single dried blood spot. In rare circumstances where a dried blood spot was not completely filled, six $3 \mathrm{~mm}$ discs were punched from multiple spots. Then, the discs were incubated with $1000 \mu \mathrm{L}$ of elution buffer for $90 \mathrm{~min}$ on a heat shaker at $37^{\circ} \mathrm{C}$ for 
elution. Then, the eluent was concentrated with a $100 \mathrm{kDa}$ molecular weight cut-off column (MWCO) for $8 \mathrm{~min}$ at $14,000 \mathrm{rcf}$.

For the testing, the eluent was assayed using a modified Antibody Detection by Agglutination-PCR (ADAP) protocol $^{15-18}$. Briefly, $8 \mu \mathrm{L}$ of eluent was incubated with $8 \mu \mathrm{L}$ of 1 femtomole of SARS-CoV-2 spike protein S1 subunit-DNA conjugate pair mixtures at $37^{\circ} \mathrm{C}$ for $30 \mathrm{~min}$. The production of the $\mathrm{S} 1$ protein-DNA conjugates was described previously ${ }^{15}$. If present, the SARS-CoV-2 antibodies in the specimen engage with S1-DNA conjugate to agglutinate into a dense immune complex. To quantify the degree of agglutination, $116 \mu \mathrm{L}$ of ligation solution $(20 \mathrm{mM}$ Tris, $50 \mathrm{mM} \mathrm{KCl}, 20 \mathrm{mM} \mathrm{MgCl} 2,20 \mathrm{mM}$ DTT, $25 \mu \mathrm{M} \mathrm{NAD}, 0.025 \mathrm{U} / \mu \mathrm{L}$ ligase, $100 \mathrm{nM}$ connector) was mixed with $4 \mu \mathrm{L}$ eluent/conjugate mixture and incubated at $30^{\circ} \mathrm{C}$ for $15 \mathrm{~min}$. Then, $25 \mu \mathrm{L}$ of the ligated solution was mixed with PCR master mix that contained primer pairs and polymerase for amplification under standard thermocycling conditions $\left(95^{\circ} \mathrm{C}\right.$ for $10 \mathrm{~min}, 95^{\circ} \mathrm{C}$ for $15 \mathrm{~s}, 56^{\circ} \mathrm{C}$ for $\left.30 \mathrm{~s}, 13 \mathrm{cycles}\right)$. The pre-amplified products were then quantified in a 96 -well qPCR plate. SYBR green-based qPCR was performed on a Bio-Rad CFX96 real-time PCR detection system $\left(95^{\circ} \mathrm{C}\right.$ for $10 \mathrm{~min}, 95^{\circ} \mathrm{C}$ for $30 \mathrm{~s}, 56^{\circ} \mathrm{C}$ for $\left.1 \mathrm{~min}, 40 \mathrm{cycles}\right)$. As a result of agglutination, specimens harboring high quantities of antibodies will have large quantities of amplifiable DNA, thus exhibiting a strong qPCR signal (low Ct). In contrast, samples without SARS-CoV-2 antibodies will have few DNA amplicons, thus yielding a weaker qPCR signal (high Ct). Instead of using the common cycle threshold (Ct) as readout, the ADAP assay readout $\Delta \mathrm{Ct}$ is defined as the $\mathrm{Ct}$ value of a blank control minus the $\mathrm{Ct}$ of the actual specimen. The magnitude of the $\Delta \mathrm{Ct}$ is proportional to the amplicon concentration in the PCR plate well, which in turn is proportional to the amount of antibody present in the sample. The $\Delta \mathrm{Ct}$ offers significant reproducibility since the subtraction of the blank control Ct and the sample $\mathrm{Ct}$ cancels out any potential drift across runs.

For multiplex analysis of both spike and nucleocapsid protein antibodies, both conjugates were incubated together with the dried blood spot eluent. In the pre-amplification step, DNA primers specific to both conjugates were included. Then, in the qPCR step, the pre-amplification product were added to each well of the qPCR plates containing corresponding primers to quantify spike protein and nucleocapsid protein antibodies separately.

To ensure the quality of the process, one positive and one negative control dried blood spots cards were always analyzed concurrently. The control cards went through the entire elution and testing process. The analysis was only valid if the positive control cards tested positive and the negative cards tested negative.

Statistics. Statistical analysis was performed using GraphPad Prism Version 8 (GraphPad Software). MannWhitney $U$ test was performed to determine the statistical significance of differences. A P value $<0.05$ was considered significant.

\section{Data availability}

All data generated or analyzed during this study are included in this published article.

Received: 15 June 2020; Accepted: 1 November 2020

Published online: 19 November 2020

\section{References}

1. Letko, M., Marzi, A. \& Munster, V. Functional assessment of cell entry and receptor usage for SARS-CoV-2 and other lineage B betacoronaviruses. Nat. Microbiol. 5, 562-569 (2020).

2. Wu, F. et al. A new coronavirus associated with human respiratory disease in China. Nature 579, 265-269 (2020).

3. Li, Q. Early transmission dynamics in Wuhan, China, of novel coronavirus-infected pneumonia. N. Engl. J. Med. 26, 1199-1207 (2020).

4. Tang, Y. W., Schmitz, J. E., Persing, D. H. \& Stratton, C. W. The laboratory diagnosis of COVID-19 infection: Current issues and challenges. J. Clin. Microbiol. https://doi.org/10.1128/JCM.00512-20 (2020).

5. Zou, L. et al. SARS-CoV-2 viral load in upper respiratory specimens of infected patients. N. Engl. J. Med. 382, 1177-1179 (2020).

6. Long, Q. X. et al. Antibody responses to SARS-CoV-2 in patients with COVID-19. Nat Med. 26, 845-848 (2020).

7. Amanat, F. et al. A serological assay to detect SARS-CoV-2 seroconversion in humans. Nat. Med. 26, 1033-1036 (2020).

8. Okba, N. M. A. et al. Severe acute respiratory syndrome coronavirus 2-specific antibody responses in coronavirus disease 2019 patients. Emerg. Infect. Dis. 26(7), 1478-1488 (2020).

9. Whitman, J. D. et al. Test performance evaluation of SARS-CoV-2 serological assays. MedRxiv. https://doi. org/10.1101/2020.04.25.20074856 (2020).

10. Lipsitch, M., Swerdlow, D. L. \& Finelli, L. Defining the epidemiology of COVID-19-Studies needed. N. Engl. J. Med. 382(13), 1194-1196 (2020).

11. Duan, K. et al. Effectiveness of convalescent plasma therapy in severe COVID-19 patients. Proc. Natl. Acad. Sci. USA 117(17), 9490-9949 (2020).

12. Joyner, M. et al. Early safety indicators of COVID-19 convalescent plasma in 5,000 patients. J. Clin. Investig. https://doi. org/10.1101/2020.05.12.20099879 (2020).

13. Chen, X. et al. Detectable serum SARS-CoV-2 viral load (RNAaemia) is closely associated with drastically elevated interleukin 6 (IL-6) level in critically ill COVID-19 patients. Clin. Infect. Diseases. https://doi.org/10.1101/2020.02.29.20029520v1 (2020).

14. Wang, W. et al. Detection of SARS-CoV-2 in different types of clinical specimens. JAMA 323(18), 1843-1844 (2020).

15. Karp, D. G. et al. Sensitive and specific detection of SARS-CoV-2 antibodies using a high-throughput, fully automated liquidhandling robotic system. SLAS Technol. https://doi.org/10.1177/2472630320950663 (2020).

16. Tsai, C. et al. Ultrasensitive antibody detection by agglutination-PCR (ADAP). ACS Central Sci. 2, 139-147 (2016).

17. Tsai, C. et al. Isotype-specific agglutination-PCR (ISAP): A sensitive and multiplex method for measuring allergen-specific IgE. J. Allergy Clin. Immunol. 141, 1901-1904 (2018).

18. Tsai, C. et al. Antibody detection by agglutination-PCR (ADAP) enables early diagnosis of HIV infection by oral fluid analysis. Proc. Natl. Acad. Sci. USA 115, 1250-1255 (2018).

\section{Acknowledgements}

We thank all patients, donors and health care staff who participated in this study. We thank Corleone Delaveris and Melissa Gray for assistance on execution of the study. The study is funded in part by NIH SBIR 
2R44DK110005-02 and 2RAI141118-02 to Enable Biosciences. The content is solely the responsibility of the authors and does not necessarily represent the official views of the NIH.

\section{Author contributions}

D.G.K., K.D., N.F. carried out the experimental work. D.S. revised the manuscript. P.V.R. and C.T.T. conceptualized the study, analyzed data and wrote the manuscript.

\section{Competing interests}

D.G.K., K.D., N.F., D.S., P.V.R. and C.T.T are employees of Enable Biosciences. K.D., P.V.R., N.F., D.S. and C.T.T are shareholders of Enable Biosciences. P.V.R and C.T.T are inventors of the ADAP patent licensed from University of California, Berkeley to Enable Biosciences. The ADAP assay used in this assay is a product in development. This does not alter our adherence to Scientific Reports policies on sharing data and materials.

\section{Additional information}

Supplementary information is available for this paper at https://doi.org/10.1038/s41598-020-76913-6.

Correspondence and requests for materials should be addressed to P.V.R. or C.T.

Reprints and permissions information is available at www.nature.com/reprints.

Publisher's note Springer Nature remains neutral with regard to jurisdictional claims in published maps and institutional affiliations.

(c) (i) Open Access This article is licensed under a Creative Commons Attribution 4.0 International License, which permits use, sharing, adaptation, distribution and reproduction in any medium or format, as long as you give appropriate credit to the original author(s) and the source, provide a link to the Creative Commons licence, and indicate if changes were made. The images or other third party material in this article are included in the article's Creative Commons licence, unless indicated otherwise in a credit line to the material. If material is not included in the article's Creative Commons licence and your intended use is not permitted by statutory regulation or exceeds the permitted use, you will need to obtain permission directly from the copyright holder. To view a copy of this licence, visit http://creativecommons.org/licenses/by/4.0/.

(C) The Author(s) 2020 